\title{
Radical penectomy: procedure details of an uncommonly performed procedure for carcinoma penis and review of literature
}

\author{
Kaushal Yadav ${ }^{* *}$ and Rakesh Minhas ${ }^{2}$ \\ ${ }^{1}$ Consultant Surgical Oncology, Max Institute of Cancer Care, Gurgaon, India \\ ${ }^{2}$ Resident Surgery, Max Institute of Cancer Care, Gurgaon, India
}

\begin{abstract}
Penile carcinoma is more common disease of developing world and in people with unhygienic practices. Partial and total penectomy are more commonly done surgical procedures. Radical penectomy is not required so commonly and only few reported cases are there for this procedure. We present technical details of this procedure in a case of young patient who was cured by this surgical technique. He required it because of disease involvement almost till proximal corpora cavernosa.
\end{abstract}

\section{Introduction}

Squamous cell carcinoma of penis represents approximately $0.5 \%$ of all cancers among men in United States and other developed countries. The incidence is higher i.e. around $10 \%$ among men of developing countries of Asia Africa and South America. In developing countries most of the cases present in advance stage. In urban India, the age-adjusted incidence of penile cancer ranges from 0.7-2.3 cases per 100,000 men while in rural India, it is 3 cases per 100,000 men, accounting for more than $6 \%$ of all malignancies in this population [1-3].

\section{Surgical anatomy of penis and surgical procedures for carcinoma penis}

From superficial to deep structures of penis are skin, superficial fascia, Buck's fascia, tunica albugenia, two paired corpora cavernosa, corpora spongiosum and urethra traversing in spongiosum. Glans is distal part of corpora spongiosum. At the root of penis corpora cavernosa are attached to ischiopubic ramus as crus. Bubospongiosus muscle originates from perineal membrane and surrounds the corpora spongiosum bilaterally lying in ventral midline. Fundiform ligament and suspensory ligaments support the penile root. Superficial penile arteries originate from external pudendal artery. Penile artery arises from right and left internal pudendal artery which further divides into bulbourethral, dorsal artery and cavernous branches. Buck's fascia splits to encase deep dorsal vessels [4].

Types of surgical procedures for carcinoma penis are described below (Figure 1):

- Penile conserving surgeries

- Glansectomy

- Partial Penectomy: Penis is cut approximately $2 \mathrm{~cm}$ proximal to tumor for adequate oncological control. Adequate penile stump of $2 \mathrm{~cm}$ should be preserved for erect urination.
- Total Penectomy: It is misnomer. Penis is excised at or near the suspensory ligament of the penis without removal of proximal corpora cavernosa. Total penectomy is indicated when size or location of penile carcinoma doesn't allow preservation of sufficient stump for upright voiding.

- Radical Penectomy: penis is excised with complete corporeal body excision till their origin. This procedure is not done commonly and there are only few reported cases [5].

\section{Case presentation}

35-year-old male patient with unhygienic general conditions presented with growth and ulcer over penis for 5 months. On examination he was having $12 \times 12 \times 10 \mathrm{~cm}$ proliferative fungating penile growth. Growth was involving most of shaft penis with induration reaching upto the base of penile shaft. Left testis was enlarged compared to right. Bilateral inguinal lymph nodes were enlarged, right side hard lymph nodes $3 \mathrm{X} 2 \mathrm{~cm}$ and left side firm inguinal node $1 \mathrm{X} 2 \mathrm{~cm}$. Biopsy taken from penile growth confirmed squamous cell carcinoma. MRI penis revealed growth involving whole of penile shaft and abutting the pubis. Only terminal part of corpora cavernosa was free from lesion (Figure 2). Bilateral inguinal lymph nodes were enlarged with suspicious metastasis. Staging with CECT thorax abdomen and pelvis was normal.

Patient was planned for curative surgery for carcinoma penis clinically T3N2 (stage IIIb). Radical penectomy was planned with elliptical incision around base. Fundiform and suspensory ligaments were ligated and cut. Prepubic fat was taken towards specimen side and pubic bone periosteum lifted. Periosteum of isciopubic rami was lifted till inferior border for adequate margins (Figure 3). Specimen

Correspondence to: Kaushal Yadav, MS, MCh, Consultant Surgical Oncology, Max Institute of Cancer Care, Gurgaon, India, E-mail: kaushalyadavoo7@yahoo.com

Received: February 07, 2018; Accepted: February 19, 2018; Published: February 22,2018 


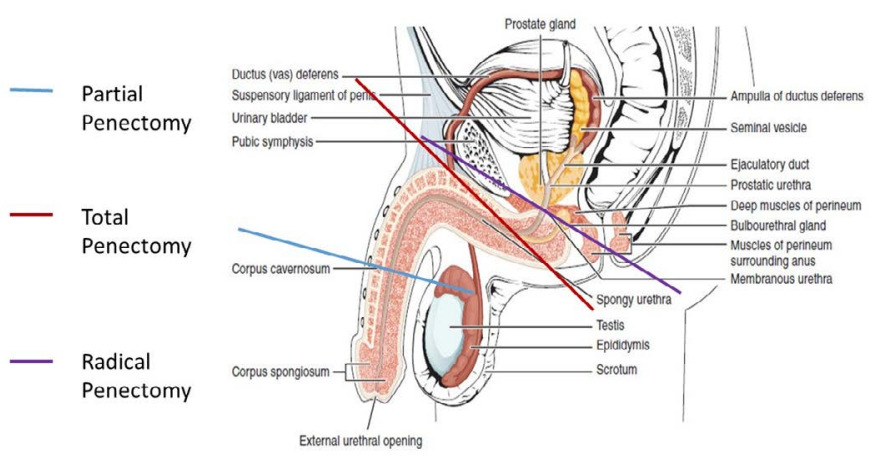

Figure 1. Extent of penile resection

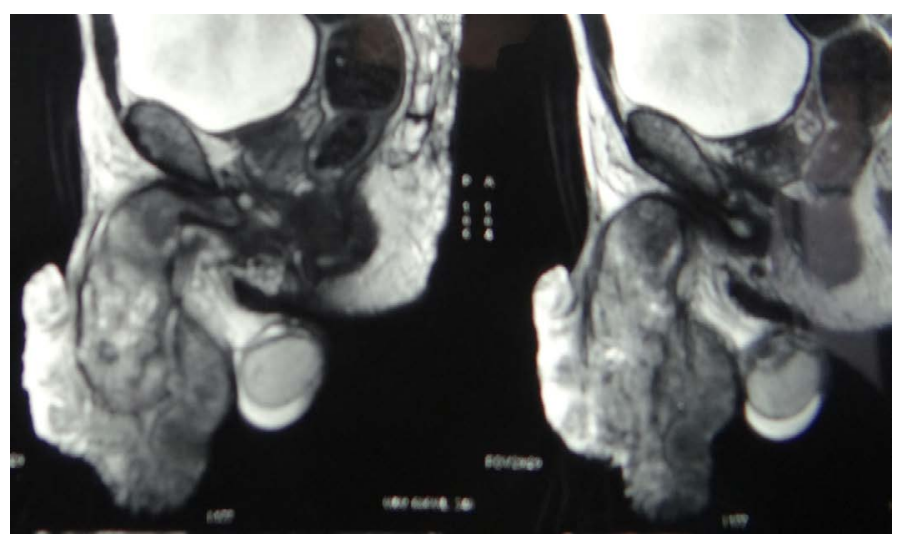

Figure 2. MRI Imaging showing: Disease till proximal crura

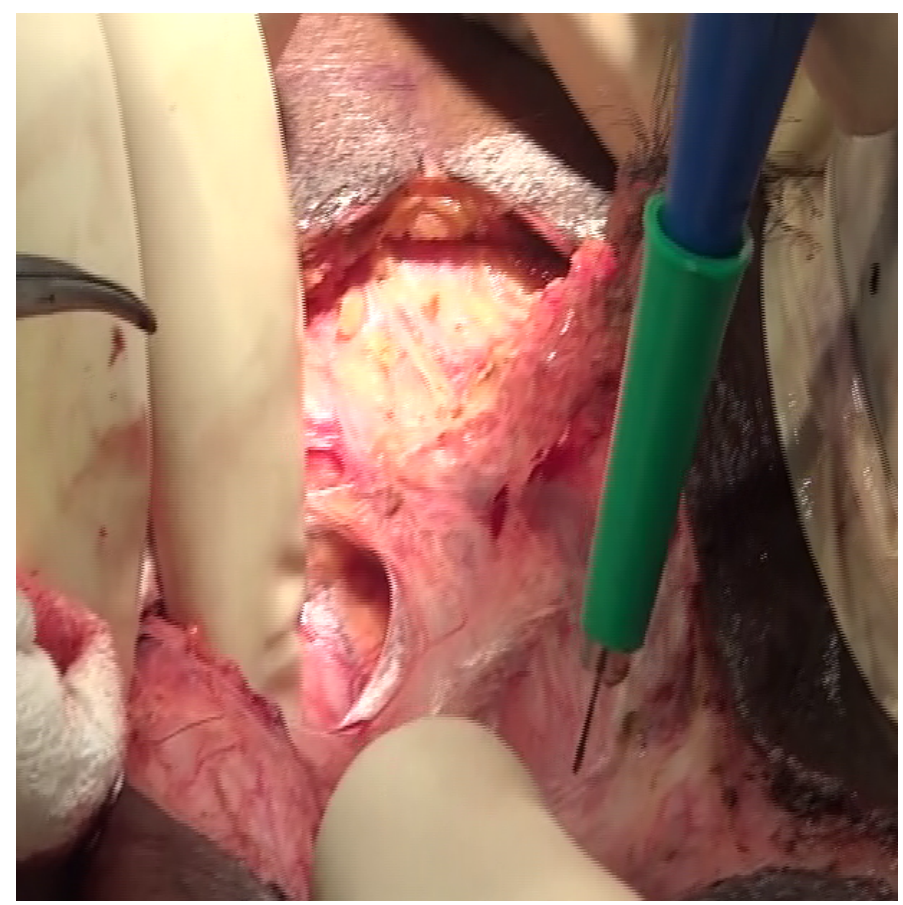

Figure 3. Intraoperative Pic: showing pubic periosteum taken along with crura

was delivered in perineum through a lambda incision in perineum. Deep dorasal vessels of penis identified ligated and cut. Left side testis was replaced with pyocele so it was excised along with the specimen. Corporeal bodies were dissected till the tip. Plane developed between proximal urethra and corpora and urethra transected leaving $3 \mathrm{~cm}$ length for perineal urothrostomy. Bilateral ilioinguinal lymph node dissection was done. Perineal urothrostomy made. Postoperative recovery was uneventful. On postoperative day 5 , he developed surgical site infection which was managed conservatively. Hisptopathology report revealed moderately differentiated squamous cell carcinoma with adequate surgical margins. Corpora cavernosa were involved while urethra was free. One right ingunal lymph node was involved with metastasis without extranodal extension i.e. pT2N1. After multidisciplinary meeting, it was decided that there is no indication of adjuvant therapy and he was kept under observation. Patient was taught self-dilatation of external meatus to avoid stenosis. At 36 months of follow-up patient was free of disease and doing his normal duty work without any problem.

\section{Discussion}

Carcinoma penis has good prognosis in early stages if adequate surgical treatment instituted, however in advanced stages it had a poor prognosis with ineffective systemic treatment options. Surgical resection with negative margins is pivotal treatment for primary. Presence of positive inguinal lymph node and extent of inguinal and pelvic lymph nodes involvement is an important prognostic factor. All primaries except $\mathrm{T} 1$, low grade, no vascular invasion and negative inguinal lymph nodes on clinical examination should undergo modified inguinal lymph node dissection and frozen section examination. Node positive frozen section or preoperative positive inguinal lymph nodes on FNAC should undergo ilioinguinal lymph node dissection [6].

In present case radical penectomy with bilateral ilioinguinal lymph node dissection was done. Postoperative histopathology report revealed moderately differentiated squamous cell carcinoma pT2N1 with negative margin status. Radical penectomy is an uncommonly performed surgery for carcinoma penis. Goal in surgical resection for carcinoma penis is to get adequate margin $2 \mathrm{~cm}$, however $5 \mathrm{~mm}$ for low grade and $1 \mathrm{~cm}$ for high grade lesion is oncologically safe. Adjuvant chemotherapy is indicating for $\mathrm{pN} 2 / \mathrm{pN} 3$ disease i.e. $\geq 2$ lymph nodes or extracapsular extension [7]. Patient was under followup surveillance postoperatively and he is disease free at 18 months of follow-up. Present case enforces the importance this surgical procedure for obtaining adequate surgical margins as shown by disease bulk.

As surgical treatment is mainstay of treatment, it is of utmost importance to obtain oncological safe negative margins. Extent of surgery increases to provide safe and healthy outcome.

\section{References}

1. Pahwa M, Girotra M, Rautela A, Abrahim R (2012) Penile cancer in India: a clinicoepidemiological study. Gulf J Oncol 12: 7-10.

2. Gopalakrishnan G (2006) Cancer penis: An overview. Ind J Urol 22: 364-367.

3. Pow-Sang MR, Ferreira U, Pow-Sang JM, Nardi AC, Destefano V (2010) Epidemiology and natural history of penile cancer. Urology 76: S2-S6.

4. Skandalakis JE, Colborn GL, Weidman TA, Foster RS Jr, et al. (2004) Skandalakis Surgical Anatomy. Chapter 25. Male Genital System.

5. Sharp DS, Angermeier KW ( $10^{\text {th }}$ edn) Surgery of Penile and Urethral Carcinoma Campbell Walsh Urology, pp: 936-940

6. Solsona E, Algaba F, Horenblas S (2004) EAU guidelines on penile cancer. Eur Urol 46: 1-8.

7. Ormsby AH, Liou LS, Oriba HA, Angermeier KW, Goldblum JR (2000) Epithelioid sarcoma of the penis: report of an unusual case and review of the literature. Ann Diag Pathol 4: 88-94. [Crossref]

Copyright: (C2018 Yadav K. This is an open-access article distributed under the terms of the Creative Commons Attribution License, which permits unrestricted use, distribution, and reproduction in any medium, provided the original author and source are credited. 